\title{
Macromolecular Rapid Communications \\ Prolate and Temperature-Responsive Self-Assemblies of Amphiphilic Random Copolymers with Perfluoroalkyl and Polyoxyethylene Side Chains in Solution --Manuscript Draft--
}

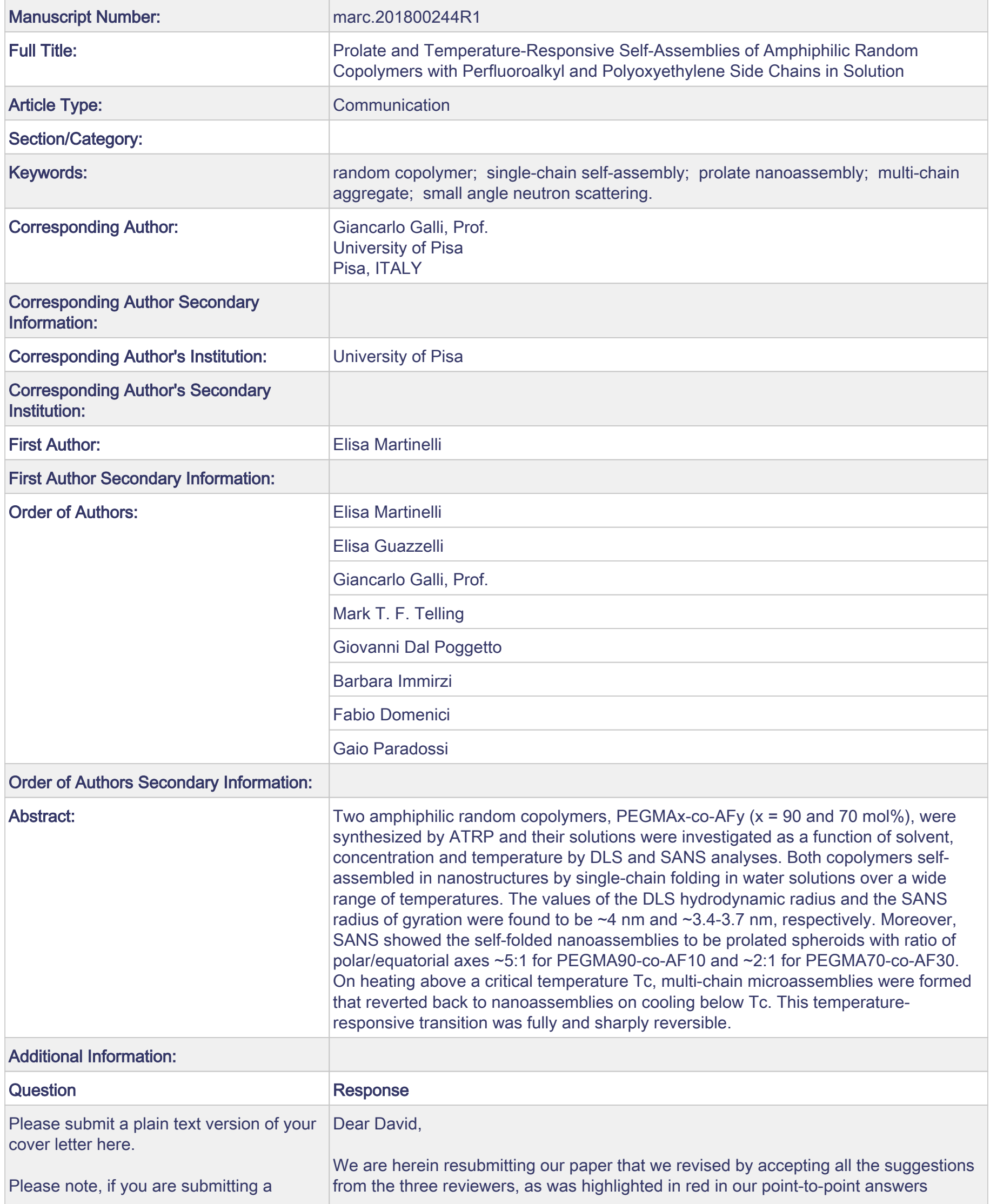


revision of your manuscript, there is an opportunity for you to provide your responses to the reviewers later; please do not add them to the cover letter.

Do you or any of your co-authors have a conflict of interest to declare? (attached).

The paper is certainly in a better shape for acceptance as a Communication in MRC and we look forward to its final acceptance.

With my best personal regards,

Giancarlo

No. The authors declare no conflict of interest. 


\section{Prolate and Temperature-Responsive Self-Assemblies of}

\section{Amphiphilic Random Copolymers with Perfluoroalkyl and}

\section{Polyoxyethylene Side Chains in Solution}

Elisa Martinelli ${ }^{1}$, Elisa Guazzelli ${ }^{1}$, Giancarlo Galli ${ }^{1 *}$, Mark T. F. Telling ${ }^{2,3}$, Giovanni Dal Poggetto ${ }^{4}$, Barbara Immirzi ${ }^{4}$, Fabio Domenici ${ }^{5}$, Gaio Paradossi ${ }^{5 *}$

${ }^{1}$ Dipartimento di Chimica e Chimica Industriale, Università di Pisa, 56124 Pisa, Italy

${ }^{2}$ STFC Rutherford Appleton Laboratory, Chilton, OX11 0QX, UK

${ }^{3}$ Department of Materials, University of Oxford, Parks Road, Oxford, UK

${ }^{4}$ Istituto per i Polimeri Compositi e Biomateriali, CNR, 80078 Pozzuoli, Italy

${ }^{5}$ Dipartimento di Scienze e Tecnologie Chimiche, Università di Roma Tor Vergata, 000133 Roma, Italy

Keywords: random copolymer; single-chain self-assembly; prolate nanoassembly; multi-chain aggregate; dynamic light scattering; small angle neutron scattering.

Two amphiphilic random copolymers, PEGMAx-co-AFy ( $x=90$ and 70 mol\%), were synthesized by ATRP and their solutions were investigated as a function of solvent, concentration and temperature by DLS and SANS analyses. Both copolymers selfassembled in nanostructures by single-chain folding in water solutions over a wide range of temperatures. The values of the DLS hydrodynamic radius and the SANS radius of gyration were found to be $\sim 4 \mathrm{~nm}$ and $\sim 3.4-3.7 \mathrm{~nm}$, respectively. Moreover, SANS showed the self-folded nanoassemblies to be prolated spheroids with ratio of polar/equatorial axes -5:1 for PEGMA90-co-AF10 and 2:1 for PEGMA70-co-AF30. On heating above a critical temperature $T_{\mathfrak{c}}$, multi-chain microassemblies were formed that reverted back to nanoassemblies on cooling below $T$ c. This temperature-responsive transition was fully and sharply reversible. 


\section{Introduction}

Special self-assembled nanostructures are generated in solution by amphiphilic copolymers, in which the hydrophilic and hydrophobic moieties interact separately and very differently with each other and with the external environment. ${ }^{[1-4]}$ In amphiphilic block copolymers, phase separation drives intermolecular self-assembly of multi-chain nano-to-macro-systems with a variety of morphologies, ranging from micelles and vesicles to continuous network structures. ${ }^{[5-8]}$ By contrast, amphiphilic random copolymers, in which the hydrophilic and hydrophobic counits are randomly distributed along the macromolecular backbone, can selffold in a selective solvent into compact single-chain coil nanoassemblies via an intramolecular self-association process. ${ }^{[9-12]}$ While the synthesis of block copolymers is generally more complicated and time-consuming, the synthesis of random copolymers is easier, as they are typically obtained by the copolymerization of two or more monomers in a one-step procedure. Moreover, current available reversible deactivation radical polymerization (RDRP) methods enable tailored syntheses of random copolymers with high fidelity of designed structural parameters to mediate solution self-assembly. ${ }^{[13,14]}$

Typical precursors of the hydrophilic component of single-chain nanoassemblies have been reported to be either ionic monomers, such as 2-acrylamido-2-methylpropanesulfonate, ${ }^{[15,16]}$ or non-ionic monomers, such as poly(ethylene glycol) methyl ether methacrylate (PEGMA) ${ }^{[10,17]}$ or acrylamide (PEGAAm) ${ }^{[18]}$. Random copolymers based on the latter type of monomers have the advantage to be soluble in both water and organic solvents and to be thermally responsive in water solution, due to the occurrence of a lower critical solution temperature-type (LCST) transition. ${ }^{[19,20]}$ The introduction of fluorinated comonomer units is known to drastically affect both bulk and surface properties of a copolymer, enhancing for example its ability to spontaneously organize at low energy surfaces and interfaces, both phenomena being driven by the lowest surface energy perfluorinated moieties. ${ }^{[21-25]}$ Amphiphilic random copolymers of PEGMA with fluoroalkyl methacrylates (FMA) were recently synthesized by ruthenium- 
catalyzed living radical polymerization and shown to give rise to single-chain self-assemblies,

referred to as unimer micelles, in water and $2 H, 3 H$-perfluoropentane solutions. ${ }^{[17]}$

The synthesis of copolymers with controlled and well-defined chemical structures may open new possibilities to construct water compatible structures by single-chain folding in which specialized nanocompartments would allow for unique activities and functions. ${ }^{[26-29]}$ Therefore, there is a growing interest in understanding the capabilities of amphiphilic random copolymers to self-assemble in different structures in solution. Along this direction, more detailed information is needed on the influence of the random copolymer structure (composition, length, sequence) on the shape of the intramolecular nanostructures in water and the role of LCST in triggering a transition to complex intermolecular assemblies.

On this basis, in this work we synthesized amphiphilic random copolymers, PEGMAx-co-FAy, composed of hydrophilic PEGMA and hydrophobic -and lipophobic- perfluorohexylethyl acrylate (FA) counits, by copper-based atom transfer radical polymerization (ATRP). Both PEGMA and FA homopolymers have low glass transition temperatures $\left(T_{\mathrm{g}}\right)$ and their copolymers appeared well suited for self-assembling in solution, notably when devising soft, low $T_{\mathrm{g}}$ and hydrophobic-core nanoobjects to be created/disrupted at a critical temperature. By combined dynamic light scattering (DLS) and small angle neutron scattering (SANS) measurements we proved the formation of single-chain nanoassemblies in solutions of different solvents over wide temperature ranges. SANS measurements also showed that the nanoassemblies were prolated ellipsoids with large ratios of polar/equatorial axes in water solutions. Such new shapes enlarge the diversity of single-chain folded nanostructures, that are commonly assumed to be compact and spherical. Moreover, these intramolecular nanostructures aggregated into intermolecular microscaled structures at a critical temperature $T_{\mathrm{c}}$, that in turn depended on the content of PEGMA counits.

\section{Experimental part}




\subsection{Materials}

Anisole (Sigma Aldrich) was vacuum distilled over sodium. CuBr (Sigma Aldrich) and ethyl $\alpha$ bromoisobutyrate (EBIB, Sigma Aldrich) were extracted with glacial acetic acid and then washed with diethyl ether, dried and stored under nitrogen. $N, N, N^{\prime}, N^{\prime \prime}, N^{\prime \prime \prime}$ Pentamethyldiethylenetriamine (PMDETA, Sigma Aldrich) was freshly distilled before use. Perfluorohexylethyl acrylate (FA, Fluoryx) and poly(ethylene glycol) methyl ether methacrylate (PEGMA, $M_{\mathrm{n}}=300 D P_{\mathrm{n}} \sim 4, D=1.2$, Sigma Aldrich) were filtered on basic alumina to remove inhibitors. Other reagents and solvents for syntheses (Sigma Aldrich) were used as received.

Synthesis of polymers. The copolymers PEGMAx-co-FAy were prepared and purified following a same ATRP procedure that is herein described in detail for PEGMA90-co-FA10. In a typical preparation, PEGMA (2.49 $\mathrm{mL}, 8.7 \mathrm{mmol})$, FA (0.418 g, $1.0 \mathrm{mmol})$, PMDETA (20.88 $\mu \mathrm{L}, 0.1 \mathrm{mmol})$, EBIB $(14.68 \mu \mathrm{L}, 0.1 \mathrm{mmol})$ and anisole $(6 \mathrm{~mL})$ were degassed in a Schlenk tube with three freeze-pump-thaw cycles. Then, $\mathrm{CuBr}(14.34 \mathrm{mg}, 0.1 \mathrm{mmol})$ was added and three more freeze-pump-thaw cycles were performed before the polymerization was started at $90{ }^{\circ} \mathrm{C}$ under nitrogen atmosphere. After $24 \mathrm{~h}$, the reaction was stopped by exposure to oxygen and quenching to $0{ }^{\circ} \mathrm{C}$. The crude product was filtered on basic alumina to remove catalyst and repeatedly precipitated from chloroform solutions into $n$-hexane ( $89 \%$ yield). The copolymer contained 90 mol\% PEGMA and 10 mol\% FA counits $\left(M_{\mathrm{n}}=34300, D=1.89\right)$. This sample was hereafter named PEGMA90-co-FA10.

${ }^{1} \mathrm{H}$ NMR $\left(\mathrm{CHCl}_{3}\right): \delta(\mathrm{ppm})=7.2-7.4$ (arom), 4.0-4.6 $\left(\mathrm{COOCH}_{2}\right), 3.4-3.6\left(\mathrm{OCH}_{2} \mathrm{CH}_{2}\right.$, $\mathrm{CHCOO}), 3.34\left(\mathrm{OCH}_{3}\right), 2.5\left(\mathrm{CH}_{2} \mathrm{CF}_{2}\right), 0.7-2.3\left(\mathrm{CH}, \mathrm{CH}_{2}, \mathrm{CH}_{3}\right)$.

${ }^{19} \mathrm{~F} \mathrm{NMR}\left(\mathrm{CHCl}_{3} / \mathrm{CF}_{3} \mathrm{COOH}\right): \delta(\mathrm{ppm})=-5.6\left(\mathrm{CF}_{3}\right),-38.5\left(\mathrm{CF}_{2} \mathrm{CH}_{2}\right),-46.0$ to $-48.1\left(\mathrm{CF}_{2}\right),-$ $51.0\left(\mathrm{CF}_{2} \mathrm{CF}_{3}\right)$.

The copolymer containing 70 mol\% PEGMA and 30 mol\% FA counits $\left(M_{\mathrm{n}}=28900, \nexists=1.43\right)$ was hereafter named PEGMA70-co-FA30. 
The reactivity ratios of PEGMA/AF6 comonomers were determined by carrying out freeradical copolymerizations using AIBN as an initiator $(1 \mathrm{wt} \%)$ in anisole solution at $90{ }^{\circ} \mathrm{C}$ for 5 min (conversion $<\sim 5-10 \%$ by ${ }^{1} \mathrm{H}$ NMR). The initial feed PEGMA/AF6 ratio was varied in the range $90 / 10-30 / 70 \mathrm{~mol} / \mathrm{mol}$.

\subsection{Methods}

${ }^{1} \mathrm{H}$ NMR and ${ }^{19} \mathrm{~F}$ NMR solution spectra were recorded with a Bruker Advance DRX 400 spectrometer.

Values of absolute average molecular weights $\left(M_{\mathrm{n}}, M_{\mathrm{w}}\right)$ were determined by size exclusion chromatography (SEC) measurements with a GPC Max Malvern instrument equipped with a Viscotek TDA 305 apparatus with three detectors (refractive index, detection angle at $90^{\circ}$ and low angle $\left(7^{\circ}\right)$ laser light scattering, viscometer). Copolymer solutions $\left(5 \mathrm{mg} \mathrm{mL}^{-1}, 0.22 \mu \mathrm{m}\right.$ PTFE-filtered for organic solutions and nylon-filtered for water solutions) in tetrahydrofuran, chloroform, dimethylformamide (10 mM LiBr), and water/methanol (90/10 v/v) were used (for more details, see Supporting Information). Chloroform solutions were also analyzed with a Jasco PU-2089Plus liquid chromatograph equipped with two PL gel $5 \mu \mathrm{m}$ mixed-D columns, a Jasco RI-2031Plus refractive index detector and a Jasco UV-2077Plus UV/vis detector for comparisons.

Dynamic light scattering (DLS) measurements were performed with a Malvern NanoZetaSizer apparatus equipped with a $5 \mathrm{~mW}$ HeNe laser (Malvern Instruments) and Peltier temperature control system ranging from $0.0{ }^{\circ} \mathrm{C}$ to $90.0{ }^{\circ} \mathrm{C}\left(0.1{ }^{\circ} \mathrm{C}\right.$ accuracy). This system uses quasibackscatter detection, i.e. the scattered light is collected at an angle of $173^{\circ}$, the backscattering being less sensitive to multiple scattering effects. In order to obtain the size distributions, the measured autocorrelation functions were analyzed by using the CONTIN algorithm. Decay times were used to determine the distribution of the diffusion coefficients $D$ of the particles, which in turn were converted to a distribution of apparent hydrodynamic diameters $d_{\mathrm{h}}$ using the 
Stokes-Einstein relationship $d_{\mathrm{h}}=k_{\mathrm{B}} T / 3 \pi \eta D$, where $k_{\mathrm{B}} T$ is the thermal energy and $\eta$ the solvent viscosity. The reported values of $d_{\mathrm{h}}$ were the average of several measurements, obtained from intensity weighted distributions. Polydispersity index (PDI) was extracted from cumulant analysis.

Eye-clear, transparent copolymer solutions (5, 2, and $0.5 \mathrm{mg} \mathrm{mL} \mathrm{m}^{-1}$ concentration) were prepared at room temperature by dissolution in MilliQ water (18.2 Mohm·cm resistivity), acetone (analytical grade, Sigma Aldrich), and trifluorotoluene ( $\geq 99 \%$, Sigma Aldrich). All solvents were filtered (Millipore filters) before use to avoid sample contamination, and then checked by DLS. For each temperature, the typical acquisition parameters were $10 \mathrm{~s}$ integration time and 30 repetitions. A delay time of 5 min was evaluated as appropriate to ensure the thermal equilibrium and the stabilization of the time dependent intensity fluctuations was reached before starting measurements. For each temperature the measurements were performed at least 3 times, and several independent experiments were performed to assess the reproducibility of results.

Small angle neutron scattering (SANS) experiments were carried out at the ISIS Spallation Neutron Source (STFC Rutherford Appleton Laboratory, Didcot, UK) using the SANS2D instrument. The experiments were performed at 25.0, 55.0 (PEGMA70-co-FA30) and $65.0{ }^{\circ} \mathrm{C}$ (PEGMA90-co-FA10) on $\mathrm{D}_{2} \mathrm{O}$ solutions $\left(2 \mathrm{~mL}^{-1}\right.$ concentration) in $2 \mathrm{~mm}$ Hellma quartz cells placed in a thermally controlled sample holder rack. An incident wavelength range of $1.75-$ $16.5 \AA$ with an instrument set up of $\mathrm{L} 1=4 \mathrm{~m}, \mathrm{~L} 2=4 \mathrm{~m}$ and a beam diameter of $8 \mathrm{~mm}$, was used resulting in an effective $Q$ range of $\sim 0.005-0.7 \AA^{-1}$. Each raw scattering data set was corrected for detector efficiency, sample transmission and solvent background scattering using the Mantid Software, and then converted to absolute scattering cross section $\left(I(Q)\left(\mathrm{cm}^{-1}\right)\right)$ as a function of momentum transfer $(Q)$ using the scattering from a standard sample in accordance with established procedures. ${ }^{[30]}$ Pure deuterated water (scattering length density $\rho_{\mathrm{D} 2 \mathrm{O}}=$ 
$6.330 \cdot 10^{10} \mathrm{~cm}^{-2}$ ) was used to provide the necessary contrast. For data analysis and fitting, the SasView software was used.

\section{Results and discussion}

Amphiphilic random copolymers, named PEGMAx-co-FAy ( $x=90$ and 70 mol\% PEGMA), were prepared by controlled ATRP of the hydrophilic poly(ethylene glycol) methyl ether methacrylate (PEGMA) and the hydrophobic/lipophobic perfluorohexylethyl acrylate (FA) by using ethyl $\alpha$-bromoisobutyrate (EBIB) as initiator, $\mathrm{CuBr}$ as catalyst, PMDETA as ligand and anisole as solvent at $90{ }^{\circ} \mathrm{C}$ for $24 \mathrm{~h}$ (Figure 1). While the initial monomer:initiator molar ratio was kept constant (100:1), the PEGMA:FA molar ratio in the feed was varied in order to obtain two copolymers with largely different compositions ( $y=10$ and $30 \mathrm{~mol} \%$ FA). Consistently, different characters of amphiphilicity and solubility were achieved, namely in water solutions. The evaluated monomer reactivity ratios, $r_{\mathrm{PEGMA}}=1.43$ and $r_{\mathrm{AF}}=0.88$ (Kelen-Tudos method), confirmed the occurred random incorporation of counits along the polymer backbone with a slight enrichment in PEGMA. An ATRP PEGMA homopolymer was also investigated for comparison.

Values of the absolute average molecular weights of the polymers were determined by SEC measurements with a triple detector (calibration) system and/or universal calibration in various solvents. Values of the Mark-Houwink slope $\alpha$ were $0.43-0.46$ for tetrahydrofuran or chloroform solutions, typical of random coil polymer chains in theta solvents. Lower values of $\alpha(0.37-0.39)$ were found for polymer solutions in dimethylformamide (10 mM LiBr), which suggests formation of more compact nanoassemblies than conventional coil polymer chains in this solvent.

The analyses of the PEGMA homopolymer and the two copolymers in water/methanol (90/10 v/v) gave different responses. The homopolymer showed a clear behaviour with a molecular weight value similar to that obtained with tetrahydrofuran solution, with value of Mark- 
Houwink slope $(\alpha=0.59)$ typical for a polymer in a random coil conformation. By contrast, meaningful values of molecular weights and viscometry parameters $(\alpha$ and $\log (\mathrm{K}))$ could not be obtained for the two copolymers. This can be due to the enhanced hydrophobic nature of the copolymers that rendered them poorly solvated in water/methanol $(90 / 10 \mathrm{v} / \mathrm{v})$ in these SEC conditions and highlights the existence of self-assembled structured copolymers (non-evaluable Mark-Houwink constants). No scattering nanoassemblies were detected by DLS on any polymer solutions in either chloroform or tetrahydrofuran (see below). Accordingly, average numbers of repeat counits of $\sim 99$ for PEGMA and $\sim 11$ for FA were evaluated for PEGMA90co-FA10 and $\sim 60$ for PEGMA and $~ 26$ for FA for PEGMA70-co-FA30 by combined NMR and SEC measurements.

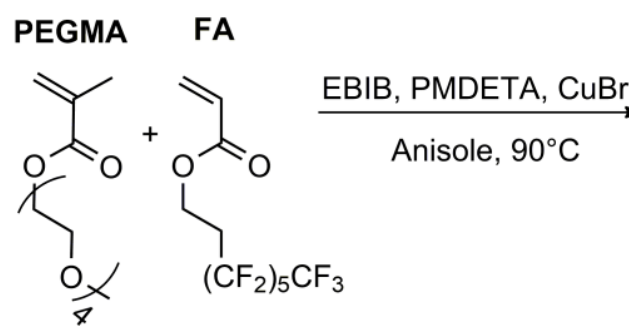

\section{PEGMAx-co-FAy}

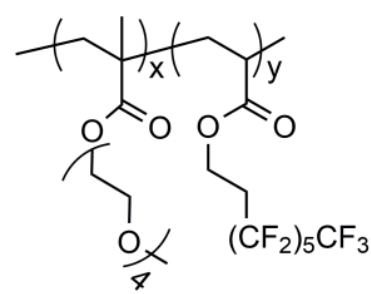

Figure 1. Schematic of the synthesis of amphiphilic random copolymers via ATRP ( $\mathrm{x}=90$ and 70 $\mathrm{mol} \%)$.

\subsection{DLS investigation of self-assembly}

DLS measurements were performed on copolymer solutions by changing solvent, concentration and temperature with heating-cooling cycles. At room temperature the solutions of both copolymers in water, chloroform, acetone and trifluorotoluene appeared clear to the eye. Average values of hydrodynamic diameter, $d_{\mathrm{h}}$, in different conditions are reported in ESI, Figure S1 and S2 and Table S1. Representative $d_{\mathrm{h}}$ values by volume percentage distribution with the corresponding trend with temperature are shown in Figure 2 for water solutions of copolymer PEGMA90-co-FA10 (for PEGMA70-co-FA30, see Figure S3 in ESI). 


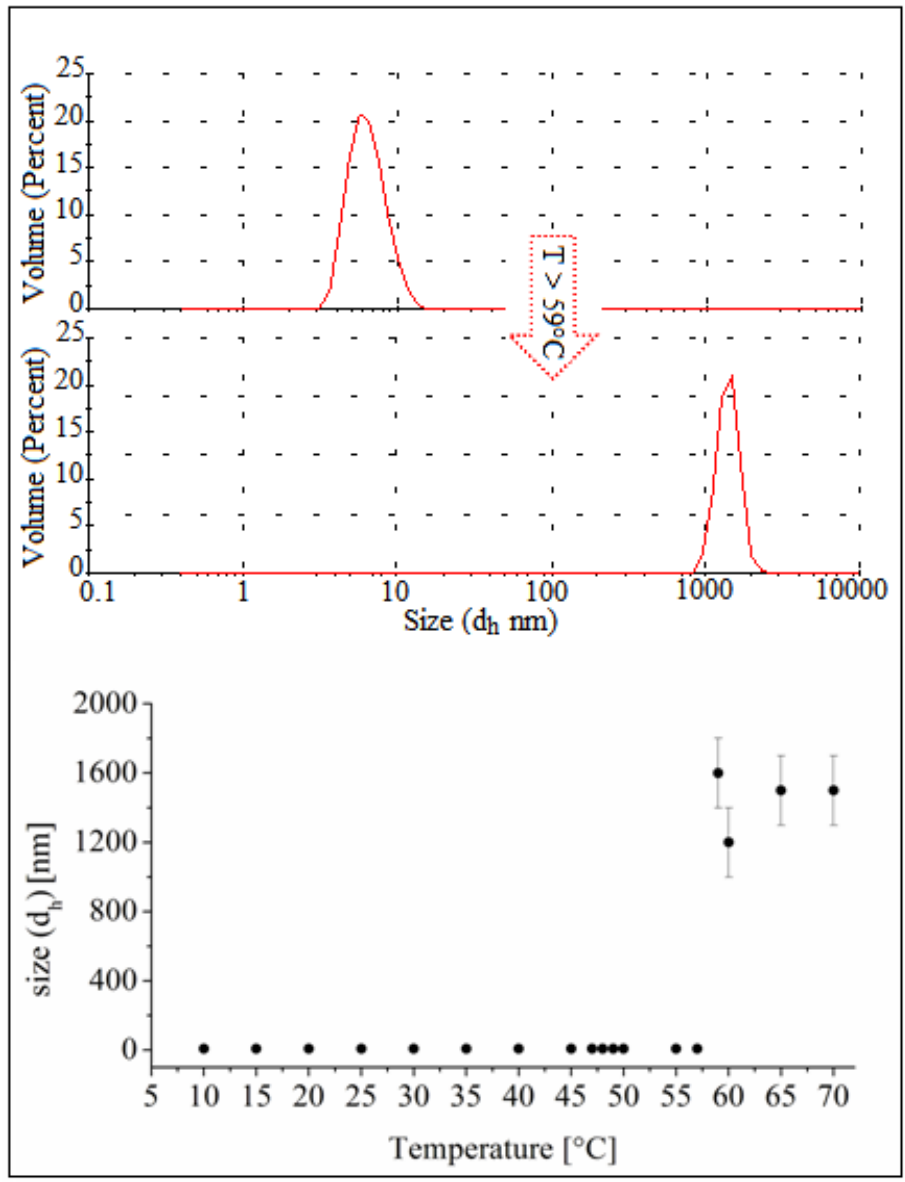

Figure 2. DLS representative $d_{\mathrm{h}}$ distributions for copolymer PEGMA90-co-FA10 in water solution (5 $\mathrm{mg} \mathrm{mL}-1$ concentration) at $25^{\circ} \mathrm{C}$ (top) and at $65^{\circ} \mathrm{C}$ (middle), and as a function of temperature (bottom) with an extrapolated critical point at $T_{\mathrm{c}}=59.0 \pm 0.5^{\circ} \mathrm{C}$.

The size distribution in water solutions was stably centered at $\sim 8 \mathrm{~nm}(\sim 0.4$ PDI), independent of the concentration and type of solvent (Figure S1 and S2 in ESI). These nanostructures were identified as nanoassemblies of single-chain folded copolymer, consistent with previous results on structurally analogous PEGMA-co-FMA copolymers. ${ }^{[17]}$ The hydrophobic interactions among the FA side chains induced self-folding in a inner, core structure of a single-chain nanoassembly, whereas the hydrophilic PEGMA side chains were exposed to contact with water in a outer, shell structure. In contrast, common synthetic polymers can fold in random coil conformations without distinct hydrophobic or hydrophilic domain structures owing to the lack of specific intramolecular interactions such as those taking place in compositionally designed amphiphilic copolymers. ${ }^{[31]}$ 
In trifluorotoluene solution, single-chain nanoassemblies with $d_{\mathrm{h}}$ of $\sim 7 \mathrm{~nm}$ and with a PDI of

$\sim 0.3$ were formed (Figure S2 in ESI). In this solvent intramolecular association of the PEGMA side chains originated reverse single-chain nanoassemblies with a core of hydrophilic side chains and a shell of hydrophobic (fluorous) side chains. Analogous reverse nanoassemblies were also found with $2 H, 3 H$-perfluoropentane solutions of PEGMA-co-FMA copolymers. ${ }^{[17]}$ A negligible population of a few hundreds $\mathrm{nm}(\sim 150-300 \mathrm{~nm})$ (Figure S4A and B in ESI) in the intensity $d_{\mathrm{h}}$ distribution was also present in water and acetone solutions, although it was not detected in the volume $d_{\mathrm{h}}$ distribution (Figure 2). However, such larger aggregates were not observed in trifluorotoluene solutions (Figure S4C in ESI). Thus, formation of larger size aggregates was induced by the intermolecular interactions between the least affine FA side chains with the water and acetone solvents. We highlight the size stability of the single-chain nanoassemblies in the presence of different solvents as a consequence of the dual affinity nature of PEGMA and FA and their random distribution along the main chain backbone.

For both copolymers in water, the size dependence on temperature exhibited a dramatic and fully reversible change in $d_{\mathrm{h}}$ at a sharp critical temperature $T_{\mathrm{c}}$ (Figure 2, Figure S1 and S3 in ESI). Above $T_{\mathrm{c}}$, the water solution turned to a turbid dispersion of larger ( $\mu \mathrm{m}$ scale), intermolecular, i.e. multi-chain, aggregates. This thermoresponsive behaviour is reminiscent of the LCST transition of PEGMA-based polymers in water solution. ${ }^{[19,20]}$ The $T_{\mathrm{c}}$ values of the two copolymers strongly depended on chemical composition. In fact $T_{\mathrm{c}}$ was $59.0 \pm 0.5{ }^{\circ} \mathrm{C}$ for PEGMA90-co-FA10 (Figure 2) but decreased to $49.0 \pm 0.5{ }^{\circ} \mathrm{C}$ for PEGMA70-co-FA30, where the content of hydrophilic PEGMA was lowered from 90 to 70 mol\% (Figure $3 \mathrm{~S}$ in ESI). No hysteresis was found in the $T_{\mathrm{c}}$ value on repeated, successive heating-cooling cycles. The size distribution was monomodal and sharp in both volume and intensity percentage distributions (Figure 2, S3 and S4A in ESI). In contrast to what was found below the respective $T_{\mathrm{c}}$, above $T_{\mathrm{c}}$ the average $d_{\mathrm{h}}$ value depended on the content of PEGMA counits, with $d_{\mathrm{h}}$ values of aggregates ranging within $\sim 0.5-\sim 1.6 \mu \mathrm{m}$ for $0.5-5 \mathrm{mg} \mathrm{mL}^{-1}$ concentrations (Table S1). Despite the 
increase in size, the PDI values were as low as $\sim 0.3$, showing a strong sharpness of size

distributions. Aggregates of both copolymers were stable in size well above their $T_{\mathrm{c}}$, indicating that the polymer aggregation process was completed (Figure 2 and S3 in ESI). Previous examples of copolymers of a longer chain PEGMA $\left(D P_{n} \sim 9\right)$ with fluoroalkyl methacrylates (PEGMA-co-FMA copolymers) and with alkyl methacrylates (PEGMA-co-RMA copolymers) underwent similar LCST transitions at considerably higher temperatures, i.e. $T_{\mathrm{c}} \sim 80-90{ }^{\circ} \mathrm{C}$ for PEGMA-co-RMA ${ }^{[11]}$ and $\sim 70-90{ }^{\circ} \mathrm{C}$ for PEGMA-co-FMA ${ }^{[17]}$. Thus, comparatively low values of $T_{\mathrm{c}}$ may be tuned for appropriately designed amphiphilic copolymers to promote better environment sensitivity and thermal responsiveness. ${ }^{[17,19,32,33]}$ At $T_{\mathrm{c}}$, interactions between the hydrophilic PEGMA chains are disrupted, water is expelled from the shell and hydrophobic interactions prevail resulting in collapsed chains merging into larger aggregates and microphase separation. Consistent with the occurrence of such process, no solutions of either copolymer exhibited a critical phenomenon up to the investigated maximum temperature in acetone or trifluorotoluene solutions (Figure S1 in ESI).

\subsection{SANS investigation of self-assembly}

SANS measurements from PEGMA70-co-FA30 and PEGMA90-co-FA10 solutions (2 mg mL ${ }^{1}, 100 \% \mathrm{D}_{2} \mathrm{O}$ solvent) were carried out both below and above their respective $T_{\mathrm{c}}$ (Figure 3 ). At $25{ }^{\circ} \mathrm{C}\left(T<T_{\mathrm{c}}\right)$, both data sets were well described using an analytical expression for $P(Q)$ which describes an ellipse. Fits were performed from 0.005 to $0.56 \mathrm{~A}^{-1}$ with the results suggesting that the solutions are comprised of prolated spheroids whose polar axes are larger than their equatorial axes. It should be noted that modelling was improved by including a small polydispersity contribution. For the PEGMA90-co-FA10 solution, the prolated spheroids had polar axes of $9.2 \pm 0.1 \mathrm{~nm}$ and equatorial axes of $1.7 \pm 0.1 \mathrm{~nm}$ (ratio $\sim 5: 1$ ). In contrast, PEGMA70-co-FA30 formed shorter spheroidal particles with polar axes of $6.30 \pm 0.06 \mathrm{~nm}$ and equatorial axes of $2.77 \pm 0.03 \mathrm{~nm}$ (ratio $\sim 2: 1$ ). While the more hydrophilic former copolymer 
presented a more expanded nanostructure, the more hydrophobic latter copolymer adopted a more compact nanostructure in water solution. Guinier analysis of the low- $Q$ region of the scattering law at $25{ }^{\circ} \mathrm{C}$ provided values of radius of gyration of $R_{\mathrm{g}}=3.4 \pm 0.2 \mathrm{~nm}$ and $R_{\mathrm{g}}=3.7$ $\pm 0.2 \mathrm{~nm}$ for PEGMA70-co-FA30 and PEGMA90-co-FA10, respectively. These values were in close agreement with the DLS values. PEGMA-co-FMA random copolymers were previously reported to single-chain fold in nanostructures with spherical shape (average diameter $38 \mathrm{~nm}$ from aqueous solution, by TEM), ${ }^{[17]}$ with a core-shell morphology of a compact hydrophobic/fluorous compartment in water solution. Amphiphilic random copolymers of PEGMA with a benzene-1,3,5-tricarboxamide methacrylate (BTAMA) were shown to fold intramolecularly and form single-chain polymeric nanoparticles with an asymmetric shape with large aspect ratio $\left(\sim 6-13\right.$, by SANS in $\mathrm{D}_{2} \mathrm{O}$ at $\left.25^{\circ} \mathrm{C}\right) \cdot{ }^{[34]}$ Elongation of those ellipsoidal nanoobjects was induced by secondary interactions, such as hydrogen-bonding and $\pi-\pi$ interactions along with hydrophobic effects.

Above $T_{\mathrm{c}}$, the dramatic changes in the SANS profiles (Figure 3) highlight the occurrence of an aggregation process, in agreement with the DLS findings. Precise evaluation of the size of the multi-chain aggregates was not possible using the instrument configuration (i.e., accessible spatial range) employed during the experiment. Nonetheless, these spectra could be generally described using a shape independent power law between 0.004 and $0.012 \AA^{-1}$ and an ellipsoidal shape factor $P(Q)$ from 0.044 to $0.55 \AA^{-1}$ (Figure S5 in ESI). It should be noted that the polar and equatorial values of the elliptical component above $T_{\mathrm{c}}$ remained comparable to those determined below $T_{\mathrm{c}}$. For both copolymer solutions, $I\left(Q, T>T_{\mathrm{c}}\right)$, as modelled between 0.004 and $0.012 \AA^{-1}$, was well described using a power law such that $I\left(Q, T>T_{\mathrm{c}}\right)$ exhibits a $q^{-\mathrm{n}} \sim q^{-4}$ dependence. The exponent was found to be $\mathrm{n}=3.87 \pm 0.04$ for PEGMA70-co-FA30 and $\mathrm{n}=$ $3.85 \pm 0.03$ for PEGMA90-co-FA10. This suggests the formation of aggregates with a smooth surface. 
Figure 3. SANS spectra and the corresponding best fits to ellipsoidal model of $\mathrm{D}_{2} \mathrm{O}$ solutions $(2 \mathrm{mg}$ $\mathrm{mL}^{-1}$ ) of copolymers: (A) PEGMA70-co-FA30 and (B) PEGMA90-co-FA10, measured below, i.e. 25 ${ }^{\circ} \mathrm{C}$ ( $\circ$ data point, — best fit), and above, i.e. $65{ }^{\circ} \mathrm{C}$ ( $\bullet$ data point, — best fit), the transition temperature $T_{\mathrm{c}}$. Errors, always smaller than the used symbols, are visible in the empty circles.

\section{Conclusions}

The two amphiphilic random copolymers PEGMAx-co-FAy formed single-chain nanoassemblies in water solution with an elongated, ellipsoidal shape with different ratios of polar/equatorial axes. This finding adds to the generally assumed spherical shape for singlechain nanoassemblies and points to an enriched scenario of existing soft nanoobjects from selfassembling (co)polymers. Thus, the questions of morphology and conformation of single-chain nanoassemblies appear to be of general relevance and require more comprehension of the roles that self-assembly shape, size and dynamics can play in order to move towards the use of tailormade amphiphilic (co)polymers as candidates for functional systems. Moreover, the two copolymers presented a manifold self-assembly capacity in water, by which intramolecular nanoassemblies sharply and reversibly collapsed to intermolecular, multi-chain aggregates (on heating) at a composition-dependent critical temperature. The precision synthesis of 
copolymers with specific macromolecular design is therefore anticipated to reflect in diverse aggregation modes, especially as a function of temperature.

\section{Acknowledgements}

Work performed with partial financial support from the University of Pisa (fondi Progetti di Ricerca di Ateneo, PRA_2017_17). We would like to thank Najet Mahmoudi (STFC) for help with the SANS measurements and subsequent analysis.

[1] J. A. Pomposo (Ed.), Single-Chain Polymer Nanoparticles Wiley-VCH, Weinheim, 2017.

[2] D. Wu, Y. Huang, F. Xu, Y. Mai, D. Yan, J. Polym. Sci., Part A: Polym. Chem. 2017, 55, 1459.

[3] X. Fan, Z. Li, X. J. Loh Polym. Chem. 2016, 7, 5898.

[4] A. O. Moughton, M. A. Hillmyer, T. P. Lodge, Macromolecules 2012, 45, 2.

[5] J. Zhang, X.-F. Chen, H.-B. Weia, X.-H. Wan, Chem. Soc. Rev. 2013, 42, 9127.

[6] W. Lulu, H. Haiying, H. Tianbai, Prog. Chem. 2014, 26, 810.

[7] A. Rosler, G. W. M. Vandermeulen, H. A. Klok, Adv. Drug Delivery Rev. 2012, 64, 270.

[8] S. J. Holder, N. A. J. M. Sommerdijk, Polym. Chem. 2011, 2, 1018.

[9] M. Matsumoto, T. Terashima, K. Matsumoto, M. Takenaka, M. Sawamoto, J. Am. Chem. Soc. 2017, 139, 7164.

[10] Y. Koda, T. Terashima, M. Sawamoto, H. D. Maynard, Polym. Chem. 2015, 6, 240.

[11] T. Terashima, T. Sugita, K. Fukae, M. Sawamoto, Macromolecules 2014, 47, 589.

[12] G. M. Ter Huurne, L. N. J. de Windt, Y. Liu, E. W. Meijer, I. K. Voets, A. R. A. Palmans, Macromolecules 2017, 50, 8562 .

[13] L. Li, K. Raghupathi, C. Song, P. Prasad, S. Thayumanavan, Chem. Commun. (Camb) 2014, 50, 13417.

[14] Y. S. Huang, J. K. Chen, T. Chen, C. F. Huang, Polymers 2017, 9, 231.

[15] Y. Morishima, S. Nomura, T. Ikeda, M. Seki, M. Kamachi, Macromolecules 1995, 28, 2874.

[16] H. Yamamoto, M. Mizusaki, K. Yoda, Y. Morshima, Macromolecules 1998, 31, 3588.

[17] Y. Koda, T. Terashima, M. Sawamoto, Macromolecules 2016, 49, 4534.

[18] Y. Kimura, T. Terashima, M. Sawamoto, Macromol. Chem. Phys. 2017, 218, 10.1002/macp.201700230.

[19] J.-F. Lutz, J. Polym. Sci., Part A: Polym. Chem. 2008, 46, 3459.

[20] D. Szweda, R. Szweda, A. Dworak, B. Trzebicka, Polimery 2017, 62, 298.

[21] E. Martinelli, I. Del Moro, G. Galli, M. Barbaglia, C. Bibbiani, E. Mennillo, M. Oliva, C. Pretti, D. Antonioli, M. Laus, ACS Appl. Mater. Interfaces 2015, 7, 8293.

[22] E. Martinelli, A. Glisenti, B. Gallot, G. Galli, Macromol. Chem. Phys. 2009, 210, 1746.

[23] E. Martinelli, C. Fantoni, G. Galli, B. Gallot, A. Glisenti, Mol. Cryst. Liq. Cryst. 2009, 500, 51.

[24] E. Martinelli, E. Guazzelli, C. Bartoli, M. Gazzarri, F. Chiellini, G. Galli, M. E. Callow, J. A. Callow, J. A. Finlay, S. Hill, J. Polym. Sci., Part A: Polym. Chem. 2015, 53, 1213.

[25] G. Martini, E. Martinelli, G. Ruggeri, G. Galli, A. Pucci, Dyes Pigm. 2015, 113, 47.

[26] O. Altintas, C. Barner-Kowollik, Macromol. Rapid Commun. 2012, 33, 958.

[27] O. Altintas, C. Barner-Kowollik, Macromol. Rapid Commun. 2016, 37, 29.

[28] Y. Azuma, T. Terashima, M. Sawamoto, ACS Macro Lett. 2017, 6, 830.

[29] T. Terashima, T. Mes, T. F. A. De Greef, M. A. J. Gillissen, P. Besenius, A. R. A. Palmans, E. W. Meijer, J. Am. Chem. Soc. 2011, 133, 4742.

[30] G. D. Wignall, F. S. Bates, J. Appl. Cryst. 1987, 20, 28.

[31] J. A. Pomposo, I. Perez-Baena, F. Lo Verso, A. J. Moreno, A. Arbe, J. Colmenero, ACS Macro Lett. 2014, 3, 767. 
[32] E. Martinelli, E. Guazzelli, E. Masotti, T. Biver, A. Pucci, G. Galli, J. Polym. Sci., Part A: Polym. Chem. 2018, 56, 797.

[33] E. Martinelli, L. Annunziata, E. Guazzelli, A. Pucci, T. Biver, G. Galli, Macromol. Chem. Phys. 2018, 219, 10.1002/macp.201800082.

[34] P. J. M. Stals, M. A. J. Gillissen, T. F. E. Paffen, T. F. A. de Greef, P. Lindner, E. W. Meijer, A. R. A. Palmans, I. K. Voets, Macromolecules 2014, 47, 2947. 


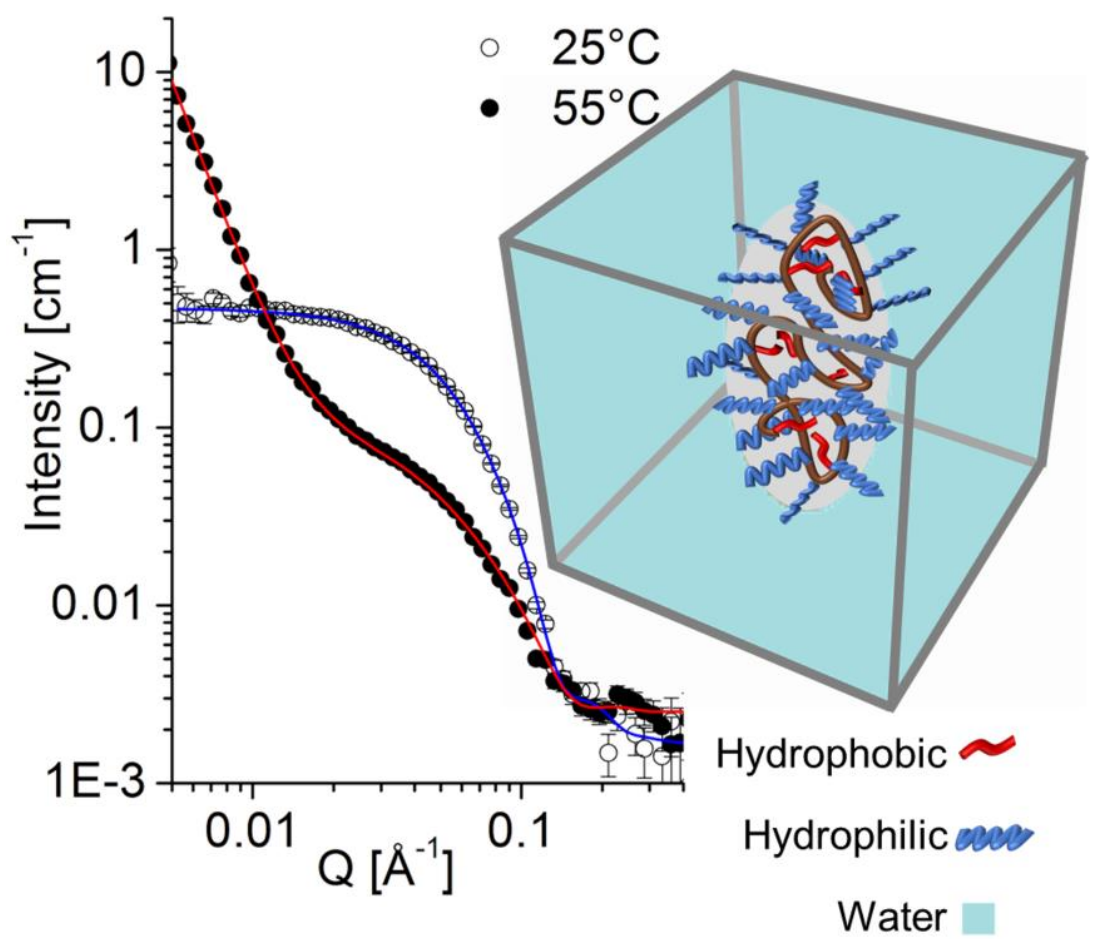

DLS and SANS analyses of ATRP amphiphilic random copolymers show the existence of nanoassemblies via spontaneous single-chain folding in water and other solvents over a wide temperature range. The nanoassemblies are prolated spheroids in $\mathrm{D}_{2} \mathrm{O}$. At a critical temperature, multi-chain microassemblies are formed that fully and sharply revert back to single-chain nanoassemblies. 
Click here to access/download

\section{Production Data \\ Production data.docx}

